\title{
A REFINEMENT OF THE KOOI'S INEQUALITY, MITTENPUNKT AND APPLICATIONS
}

\section{MARTIN LUKAREVSKi AND DAN STEFAN MARINESCU}

Abstract. Kooi's inequality is important in the theory of triangle inequalities, giving bound on the semiperimeter in terms of circumradius and inradius. We give one refinement, then relate the inequality to the Mittenpunk, and finally apply it to improve several known inequalities.

Mathematics subject classification (2010): 51M04, 51M16. equality.

Keywords and phrases: Kooi's inequality, Mittenpunkt, barycentric coordinates, Hadwiger-Finsler in-

\section{REFERENCES}

[1] O. Bottema, R. Z. Djordjevic, R. R. Janic, D. S. Mitrinovic, P. M. Vasic, Geometric inequalities, Groningen, Wolters-Noordhoff, 1969.

[2] T. R. CURry, The American Mathematical Monthly, 73 (1966), no. 2, Problem E 1861.

[3] P. Finsler, H. Hadwiger, Einige Relationen im Dreieck, Commentarii Mathematici Helvetici, 10 (1937), no.1, 316-326.

[4] Problem 825 (proposed by J. Garfunkel, solution by L. Bankoff), Crux Math. 9 (1983), 79 and 10 (1984), 168.

[5] C. Kimberling, Central Points and Central Lines in the Plane of a Triangle, Math. Mag. 67 (1994), no. $3,163-187$.

[6] O. KooI, Inequalities for the triangle, Simon Stevin, 32 (1958), 97-101.

[7] M. LuKarevs Ki, An alternate proof of Gerretsen's inequalities, Elem. Math. 72, No. 1 (2017), 2-8.

[8] M. Lukarevs Ki, The American Mathematical Monthly, 123, (2016), no. 9, Problem 11938.

[9] M. LukAREVSKI, The excentral triangle and a curious applications to inequalities, The Math. Gazette 102, No. 555, (November 2018), 531-533.

[10] D. S. Marinescu, M. Monea, M. Opincariu, M. Stroe, Note on Hadwiger-Finsler's inequalities, J. Math. Inequal. 6, No. 1, 57-64 (2012).

[11] D. S. MitrinoviĆ, J. PeČArić, V. Volenec, Recent Advances in Geometric Inequalities, Kluwer Academic Publishers, Dordrecht (1989).

[12] Ramus-Rouché, Question 233, Nouv. Ann. Math. 10 (1851), 353.

[13] V. Volenec, Metrical relations in barycentric coordinates, Math. Commun. 8, No. 1, 55-68 (2003).

[14] S. WU, A sharpened version of the fundamental triangle inequality, Math. Inequal. Appl. 11 (2008), no. 3, 477-482.

[15] S. Wu, L. Debnath, Parametrized Garfunkel-Bankoff inequality and improved Finsler-Hadwiger inequality, Appl. Math. Lett. 25, No. 3, 331-336 (2010).

[16] P. YIU, The uses of homogeneous barycentric coordinates in plane Euclidean geometry, Internat. J. Math. Ed. Sci. Tech. 31 (2000), no. 4, 569-578. 